REVIEW

\title{
Respiratory management of the infant with type 1 spinal muscular atrophy
}

\author{
A Bush, J Fraser, E Jardine, J Paton, A Simonds, C Wallis
}

Arch Dis Child 2005;90:709-711. doi: 10.1136/adc.2004.065961

A recent paper has highlighted the differences in the respiratory management offered to infants with type 1 spinal muscular atrophy (SMA-1).' Current views appear polarised between those who would offer nothing, to those who would proceed as far even as tracheostomy and long term invasive ventilation for these infants. Here we offer a personal view, as a possible template for managing a vexed and emotional problem. The complex nonrespiratory aspects of the holistic care of these infants will not be discussed.

See end of article for authors' affiliations .....................

Correspondence to: Prof A Bush, Department of Paediatric Respiratory Medicine, Royal Brompton Hospital, Sydney Street, London SW3 6NP, UK; a.bush@rbh.nthames. nhs.uk

Accepted 14 January 2005
S The first is that the prognosis of children with neuromuscular disease has changed dramatically with the increasing use of noninvasive respiratory support. Consequently, the older literature is now outdated, and should not be allowed to influence modern thinking unduly. For example, the prognosis of children with Duchenne muscular dystrophy with hypercapnic respiratory failure has been transformed from less than 10 months before death to survival for at least five years in two thirds of children. ${ }^{2}$ There are published reports of survival of SMA-1 children into the second decade of life with non-invasive respiratory support. ${ }^{3}$ Secondly, the "disabled" rightly refuse to accept assessments of their quality of life by the fit and healthy. Patients with Duchenne dystrophy, wheelchair bound and dependent on nasal intermittent positive pressure (NIPPV) at night, rate their quality of life in the relevant domains of generic quality of life measures such as the SF-36 as comparable to patients with chronic obstructive pulmonary disease (COPD)-and there is no debate about whether COPD patients should be treated actively, including with NIPPV when appropriate. Thirdly, there is wider access to information, particularly over the internet and through patient self-help organisations than ever before; even were it to be considered right paternalistically to withhold information from parents, it is no longer a workable strategy. Parents will find out all about the possible options for respiratory support for their child, whether professionals tell them or not; and concealment can only lead to a breakdown in trust. It follows logically that it is much better to pre-empt matters by having an open discussion with the parents about what is possible.

Each case should be considered on an individual basis. SMA-1 may be a useful label for some ome preliminary observations are pertinent. children, but there is a range of disabilities within the label, from the baby with severe paralysis of antenatal onset, to the later diagnosed infant, who may have some anti-gravity power, and who also has normal intelligence. We suggest that the primary aim of the assessment should be to determine a treatment plan electively. The goal should be to avoid making decisions in a crisis about a critically ill child who has collapsed and is brought to hospital nearly moribund.

Ideally, the child should be seen by a team of professionals with expertise in the management of type I SMA. The patient's care should neither be dependent on an individual practitioner's beliefs, nor a systematic failure of referral patterns to the appropriate tertiary centre. It is the responsibility of regional specialist commissioning groups to ensure that such operational policies are in place. Any plans need to be set in the context of the complex reality of care in the United Kingdom. The NHS is centrally funded and interventions, even complex ones, are free to families. However, it is true that there is often regional variation in care delivered. In part, this depends on how funds are allocated within each region, but in the case of SMA it will also be strongly driven by physician beliefs and experience.

\section{THE CHILD WHO IS SELF-VENTILATING}

It is much better if a child with SMA can be seen before intensive respiratory support is required and a possible treatment plan for when the inevitable respiratory failure occurs can be developed electively. The first step should be a multidisciplinary assessment of the child and family. This is likely to be expedited by doing it as an in-patient or day case. It is essential to enlist the help of an experienced nurse and physiotherapist to explain the demanding nature of the non-invasive regimens that have been espoused. ${ }^{3}$ Meeting a clinical psychologist may help the family to work through feelings of guilt, as well as help them cope with the ongoing stresses from having a child with such a complicated problem. A social worker, if available, can help to explore with the family what resources can be made available at home. If it can be arranged, many families find it helpful to talk to a family who cares for a child with SMA at home.

If the family do wish to proceed with active treatment, the next step is a multichannel

Abbreviations: NIPPV, nasal intermittent positive pressure; SMA-1, type 1 spinal muscular atrophy 
respiratory sleep study with measurement of carbon dioxide tensions. If the child has evidence of hypercapnic respiratory failure, it may be appropriate to consider nocturnal NIPPV. This may allow the respiratory muscles to rest, return ventilatory responses to normal, and enable the child to cope better without ventilation during the day. If the child does not have chronic respiratory failure when well, it may still be appropriate to provide a ventilator for short term use during exacerbations of symptoms during infective episodes. Long term daytime NIPPV in combination with nocturnal ventilation would leave the child essentially ventilator dependent, and is not a realistic long term option. If nothing else, the intrusive effects of the mask on normal development, and the likelihood that the facial skin will break down from pressure effects, preclude this option. In contrast, short term daytime use may be legitimate as an adjunct to physiotherapy, and also during infections. Children with SMA-1 may have disproportionate weakness of expiratory muscles, and coughing and secretion clearance needs to be evaluated. The components of cough are airway sensing of secretions (not usually a problem in SMA-1); a deep inspiration, which may be augmented using NIPPV; glottal closure; a forceful expiratory effort, which may be augmented by physiotherapy; and opening of the airway to expel secretions. Reversible causes of increased secretions should be sought. If the infant has not already been evaluated both for incoordinate swallowing and also for gastro-oesophageal reflux, this should be done and appropriate treatment instituted. It may be worth considering the use of the cough in/exsuffflator, a non-invasive device that augments inspiratory effort with positive pressure, and augments expiratory expulsive efforts. The interface with type I SMA patients has to be a mask not a mouthpiece. The device has been shown in older children and adults to be superior to physiotherapy and NIPPV in augmenting cough, in those with weak muscles. ${ }^{4}$ There are no long term studies documenting better outcome with this device, and it is expensive (about $€ 10000$ ), but it is probable that it will be increasingly recommended in this context. However, despite all these measures, it is likely that the child will require repeated suctioning, and appropriate apparatus should be provided.

Most patients are in diurnal respiratory failure by age 2 years, and in the first five years of life, acute deteriorations are likely, ${ }^{3}$ and a plan of management should be discussed with the parents. Short periods of intubation may be appropriate for acute deteriorations, such as with viral infection.. Extubation should be planned on a protocol driven basis and not attempted until gas exchange is normal in air. ${ }^{5}$ If the child requires repeated intubations over a short period (and this is unusual), the question of whether continued periods of positive pressure ventilation is right for the child will need review. Overall, those who survive have fewer intubations as they get older, and indeed in one series, there were no admissions in children over age 5 years who were treated with NIPPV at night, and during the daytime when they had a respiratory infection, and cough in/exsufflation. ${ }^{3}$

Summarising these discussions in a written plan for the parents provides an opportunity for misunderstandings to be corrected. Parents should know that any plans are not set in stone and that they have the right to change their minds. Nevertheless such discussion clarifies thinking and helps ensure that parents' views are understood and respected. Sometimes parents will disagree with the clinician's view or even with each other; then another opinion can be sought while there is still time, and not during a crisis.

\section{THE CHILD WHO IS ALREADY VENTILATED}

Occasionally, the respiratory paediatrician becomes involved when a child with SMA- 1 is already ventilated in an intensive care unit, and is proving difficult to wean either from the ventilator to NIPPV support, or from continuous NIPPV support alone. This commonly occurs in two situations: firstly, the infant with aggressive disease and rapid onset respiratory failure, who may have been ventilated before a diagnosis had been established; and secondly the older child with a more chronic, but still precarious, course of disease, dependent on nocturnal NIPPV, who has developed acute respiratory failure secondary to an intercurrent infection, and who has failed to recover to their previous state. Although such disease heterogeneity exists, a common approach to decision making is possible. The options would appear to be initiating NIPPV and energetic management of respiratory secretions, but with the decision that re-intubation will not be performed if this strategy fails; palliative care, in which short term continuous NIPPV may be used to bridge the child to a peaceful and dignified death, either in the PICU, hospice or home setting; and finally tracheostomy. Although tracheostomy is seen as a long term treatment option for SMA-1 in some countries, notably Japan, most paediatricians in Britain would feel that this is rarely, if ever, in the best interests of the child. One series ${ }^{5}$ suggests that tracheostomy may compromise long term survival and quality of life in type I SMA. Secondly, it is likely to make the management of secretions more difficult because of its negative impact on effective cough. It will certainly affect the infant's ability to communicate through vocalisation.

It is essential that open channels of communication are developed between the family and professionals from the outset. In order to ensure a uniform approach, it is best that a "core" group of professionals be nominated as the links between the team and the family. This group might include the respiratory consultant, a designated paediatric intensivist, and key members of the nursing and physiotherapy staff. The physicians should refrain from assuming that what they believe to be in the best interests of the child is correct, but aim instead to present an honest account of what life entails for a family caring for a child on domiciliary NIPPV. Equally, since many parents will want information about the option of a tracheostomy, it is better to have an open discussion about this, and make the case as to why such a strategy would not be in the best interests of the child. Alternatively, if a decision is taken to proceed to palliative care, the physician should also discuss what this entails in a positive manner. Regular multidisciplinary meetings with the family are helpful. It is important that the burden of decision-making is "shared" between the medical staff and family group.

Ideally, prior to the child being admitted to intensive care, an opportunity will have been provided to document clearly any advanced directives, which should be made known to ICU and emergency staff before the crisis intervenes. In most circumstances, the best outcome is to gradually wean ventilation and to extubate to a form of NIPPV, accepting that this might be prolonged and require several attempts. In view of genuine concerns relating to long term morbidity, tracheostomy should generally be avoided. ${ }^{3}$ On the rare occasion that disagreement arises between physicians and family as to the best way to proceed, a second opinion should be sought either from within the primary hospital or from an alternative institution to help reach a conclusion.

\section{CONCLUSION}

The management of children with severe SMA is a highly controversial area. We believe that, if requested, NIPPV should be offered at night-time, and during the day for short periods of exacerbation, together with efforts to augment cough. We would discourage long term day time NIPPV, and the use of tracheostomy. Many will disagree with the above approach in good faith. We believe that it offers a way to 
reconcile the interests of the child, the views of the parents, and the requirements of best medical practice in this complex area.

\section{Authors' affiliations}

A Bush, A Simonds, Royal Brompton Hospital, London, UK

J Fraser, Paediatric Intensive Care Unit, Bristol Royal Hospital for Children, Bristol, UK

E Jardine, J Paton, Division of Developmental Medicine, University of Glasgow Royal Hospital for Sick Children, Glasgow, UK

C Wallis, Great Ormond Street Hospital, London, UK

Competing interests: none declared

\section{REFERENCES}

1 Hardart MK, Truog RD. Spinal muscular atrophy-type 1. Arch Dis Child 2003;88:848-50.

2 Simonds AK, Muntoni F, Heather S, et al. Impact of nasal ventilation on survival in hypercapnic Duchenne muscular dystrophy. Thorax 1998; 53:949-52.

3 Bach JR, Baird JS, Plosky D, et al. Spinal muscular atrophy type 1: management and outcomes. Pediatr Pulmonol 2002;34:16-22.

4 Chatwin M, Ross E, Hart N, et al. Cough augmentation with mechanical insufflation/exsufflation in patients with neuromuscular weakness. Eur Respir J 2003;21:502-8.

5 Bach JR, Niranjan V, Weaver B. Spinal muscular atrophy type 1: a non-invasive respiratory management approach. Chest 2000;117:110-15.

\section{IMAGES IN PAEDIATRICS}

\section{Infantile cortical hyperostosis}

A 14 week old male infant presented with multiple tender bony swellings involving the legs, forearm, and lower jaw since 1 month of age (fig 1). No history of fever, trauma, or child abuse was forthcoming. He was irritable and had difficulty in feeding since the appearance of the jaw swelling. Bowing of the lower limbs with pseudoparalysis was observed. There was no response to adequate vitamin $\mathrm{C}$ supplements received prior to hospitalisation. Investigations showed mild increase of ESR, normal blood biochemistry, sterile cultures, and negative parental serology for syphilis. Bone radiographs revealed periosteal elevation, new bone formation, and cortical thickening involving the diaphyses of bilateral tibia, ulna, and femur (fig 2). The characteristic triad of irritability, swelling, and bone lesions, age at presentation, and mandibular involvement clinched the diagnosis.

Infantile cortical hyperostosis or Caffey's disease classically presents in infants less than 5 months of age, though has also been reported to occur in utero. Mandibular involvement is seen in $75-80 \%$. Radiological findings are diagnostic; however, other conditions with similar clinicoradiological features such as scurvy, osteomyleitis, syphilitic osteochondritis, hypervitaminosis A, metastatic neuroblastoma, and
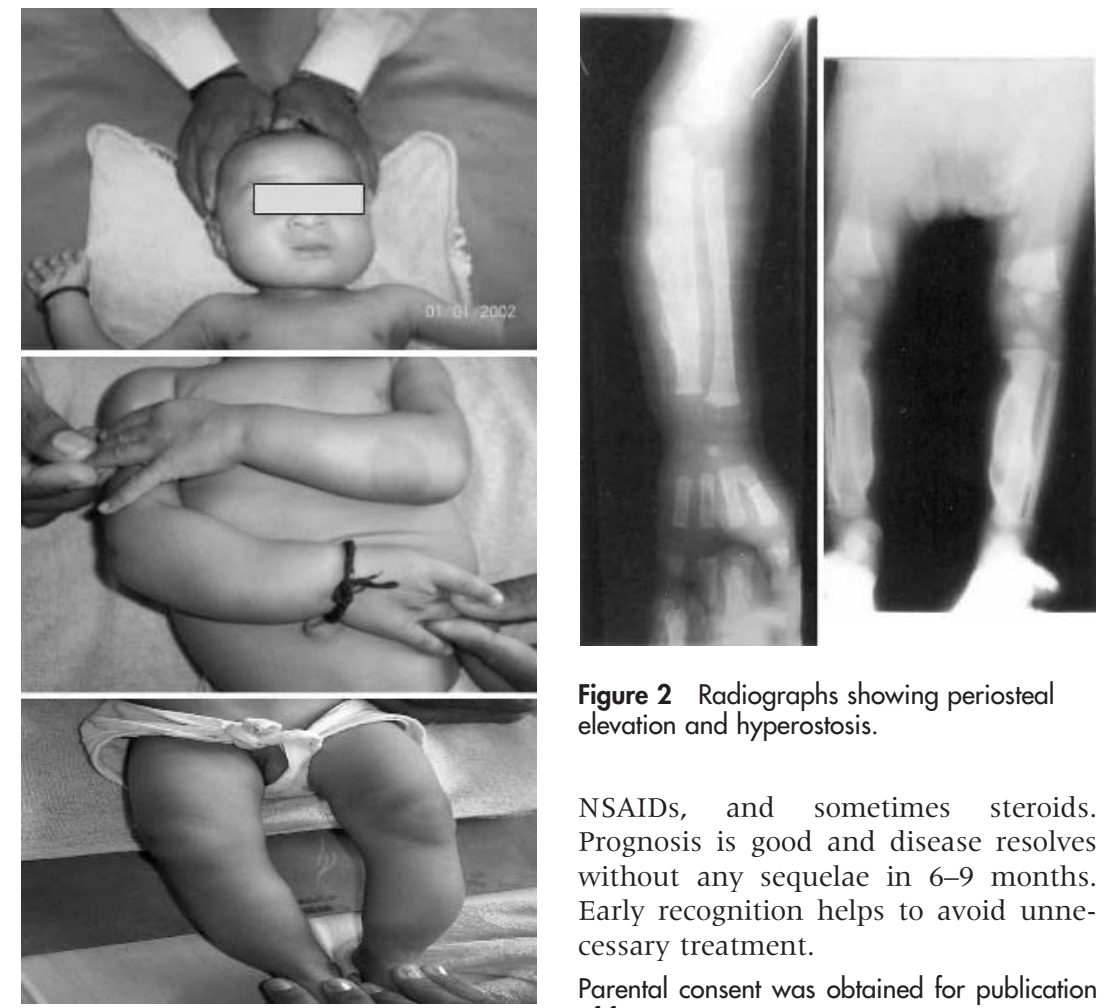

Figure 2 Radiographs showing periosteal elevation and hyperostosis.

NSAIDs, and sometimes steroids. Prognosis is good and disease resolves without any sequelae in 6-9 months. Early recognition helps to avoid unnecessary treatment.

Parental consent was obtained for publication of figure 1

D Suri, D Dayal, M Singh limbs.

battered baby syndrome need to be excluded. Treatment is palliative with
Advanced Pediatrics Center, Postgraduate Institute of Medical Education and Research, Chandigarh-160012, India; meenusingh4@rediffmail.com 